\title{
Saturable absorption dynamics of a cyanovinyl-diethylaniline dye
}

\author{
A. Penzkofer, A. Beidoun \\ Naturwissenschaftliche Fakultät II - Physik, Universität Regensburg, D-8400 Regensburg, Federal Republic of Germany
}

and

G. Wagenblast

BASF Aktiengesellschaft, D-6700 Ludwigshafen, Federal Republic of Germany

Received 16 July 1990; in final form 11 September 1990

The saturable absorption dynamics of 4-tricyanovinyl-N,N-diethylaniline (TCVA) in methanol is investigated with intense picosecond light pulses at $527 \mathrm{~nm}$. An excited-state absorption cross-section of $\sigma_{\mathrm{ex}}=(2.5 \pm 0.3) \times 10^{-17} \mathrm{~cm}^{2}$ and an absorption recovery time of $\tau_{\mathrm{A}}=3 \pm 1 \mathrm{ps}$ are determined. The fast deactivation of the $S_{1}$ state is due to internal conversion.

\section{Introduction}

Dicyanovinyl-dialkylaniline dyes [1-3] (=dialkylamino-dicyanostyrenes $=$ dialkylamino-benzylidene malonitriles) and tricyanovinyl-dialkylaniline dyes [1] (=dialkylamino-tricyanostyrenes) are applied for coloring synthetic polymer fibers [1]. They are used as sublimable dyes in heat-transfer recording materials $[4,5]$ and photoconductive recording materials [6]. They act as cytotoxic agents against tumors [7], as X-ray protective agents [8], and as photostabilizers in plastics against ultraviolet radiation [2].

The absorption and emission spectroscopic properties of 4-dicyanovinyl-N,N-diethylaniline (DCVA) [9-16] and 4-tricyanovinyl-N,N-diethylaniline (TCVA) $[15,16]$ have been investigated recently. The fluorescence quantum efficiencies $q$ in the vapor phase [15] and in liquid solutions [9-12,16] were found to be very small $\left(q<10^{-3}\right)$. Temperature-dependent fluorescence-quantum-efficiency measurements indicated an intrinsic barrierless deactivation [16]. Since the viscous drag of the dye surroundings determines the fluorescence lifetime of these dyes [9$13,17-21]$, they are applied as micro-viscosity probes and free-volume probes in micellar emulsions [9] and polymers [10-13,17-21]. The analysis of the solvent-dependent spectral absorption and emission shifts revealed a partial intramolecular charge transfer in the excited $S_{1}$ state $[9,10,13,16,18,22,23]$.

The low fluorescence quantum efficiencies may be due to fast internal conversion caused by the rotational movement of flexible molecular groups $[10,11,13,16,17,24]$. In this case, the fluorescence signal is emitted from the vibrationally relaxed excited $S_{1}$ state which has a radiative lifetime $\tau_{\text {rad }}^{\mathrm{S}_{1}}$, and the fluorescence lifetime, $\tau_{\mathrm{F}}^{\mathrm{S}_{1}}=q \tau_{\mathrm{rad}}^{\mathrm{S}_{1}}$, is equal to the absorption recovery time $\tau_{\mathrm{A}}$.

Low fluorescence quantum efficiencies could also be the result of fast intersystem crossing to triplet states. In this case, the absorption recovery time $\tau_{\mathrm{A}}$ would become equal to the phosphorescence time $\tau_{\mathrm{p}}^{\mathrm{T}_{1}}=q \tau_{\mathrm{rad}}^{\mathrm{T}_{1}}$. But the investigated dyes exhibit very little triplet yield [13], so fast intersystem crossing may be excluded.

In electron-donor-acceptor systems like the disubstituted benzenes DCVA and TCVA [10,13,24], an excited orthogonal zwitterionic singlet state might be formed [25-29] (twisted intramolecular chargetransfer state, TICT state [30-34]). The TICT state would have a long radiative lifetime $\tau_{\text {rad }}^{\text {TICT }}$ because its radiative transition to the electronic ground state is electric-dipole forbidden [30]. Therefore the fluorescence emission from TICT states would result in 
low fluorescence quantum efficiencies even at slow nonradiative decay rates. The ground-state absorption recovery time $\tau_{\mathrm{A}}$ would become equal to the TICT fluorescence lifetime $\tau_{\mathrm{F}}^{\mathrm{TICT}}=q \tau_{\mathrm{rad}}^{\mathrm{TICT}}$.

Saturable absorption studies, which measure the absorption recovery time, allow one to distinguish between internal conversion (short absorption recovery time) and TICT state formation (slow absorption recovery time).

In this paper the saturable absorption of TCVA dissolved in methanol is investigated at room temperature. The dye solutions are excited with secondharmonic picosecond light pulses of a mode-locked $\mathrm{Nd}$ :glass laser. The excited-state absorption crosssection $\sigma_{\mathrm{ex}}$ at the second-harmonic laser frequency $\left(\lambda_{\mathrm{P}}=527 \mathrm{~nm}\right)$ and the absorption recovery time $\tau_{\mathrm{A}}$ are determined by measuring the intensity dependence of transmission.

\section{Experimental}

The experimental arrangement for pump-pulse bleaching and time-delayed probe-pulse transmission measurements is displayed in fig. 1. A passively mode-locked Nd:phosphate glass laser [35] generates trains of picosecond light pulses (wavelength $\lambda_{\mathrm{L}}=1054 \mathrm{~nm}$, duration $\Delta t_{\mathrm{L}} \approx 5 \mathrm{ps}$ ). Single pulses are selected with an electro-optic shutter and are amplified in a Nd:phosphate glass amplifier (singlepulse energy up to $5 \mathrm{~mJ}$ ). The second-harmonic light

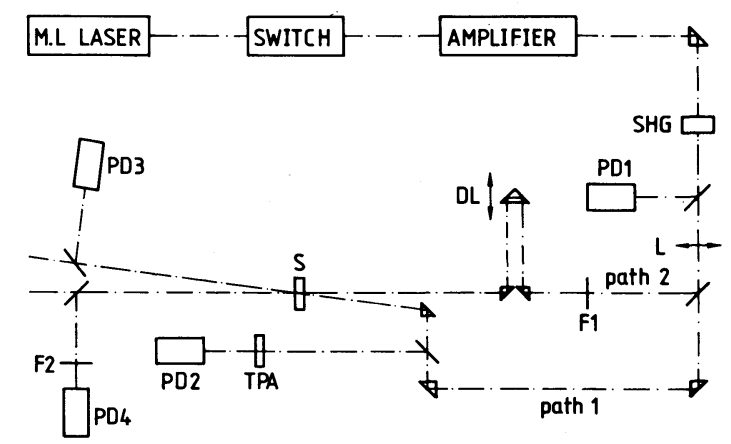

Fig. 1. Experimental setup. PD1-PD4, photodetectors, L, lens. F1, F2, filters. DL, optical delay line. SHG, KDP crystal for second-harmonic generation. TPA, rutile crystal for peak-intensity detection. S, sample. pulses (wavelength $\lambda_{\mathrm{P}}=527 \mathrm{~nm}$, duration $\Delta t_{\mathrm{P}} \approx 4 \mathrm{ps}$ ) used for the saturable absorption studies are generated in a KDP crystal. For the determination of the intensity-dependent pump-pulse transmission through the saturable absorber, only the pump pulse along path 1 is passed through the sample $S$ and the probe pulse (path 2) is covered. The input pumppulse peak intensity is determined by transmission measurements through a two-photon absorbing rutile crystal (photodetectors PD2 and PD1) [36]. The relative error of the peak-intensity detection is estimated to be $\pm 20 \%$. The pump-pulse transmission through the TCVA sample is measured with the photodetectors PD3 and PD1. The temporal behaviour of the saturable absorber at a fixed pump-pulse peak intensity is studied by measuring the transmission of a weak probe pulse (path 2) of variable delay (photodetectors PD4 and PD1). The experiments are carried out at room temperature.

\section{Results}

Intensity-dependent transmission measurements allow the determination of excited-state absorption cross-sections $\sigma_{\mathrm{ex}}$ at high pump-pulse intensities, and the determination of absorption recovery times $\tau_{\mathrm{A}}$ if they are shorter than the pump-pulse duration $\left(\tau_{\mathrm{A}} \lesssim \Delta t_{\mathrm{P}}\right)$ by comparing experimental data with numerical simulations $[37,38]$. Time-delayed probepulse transmission measurements allow the measurement of absorption recovery times if they are longer than the pump-pulse duration $\left(\tau_{\mathrm{A}}>\Delta t_{\mathrm{P}}\right)$ [39].

\subsection{Pump-pulse transmission}

The pump-pulse energy transmission $T_{\mathrm{E}}$ through TCVA in methanol versus input peak intensity $I_{0 P}$ is shown by the circles in fig. 2 (small signal transmission $T_{0}=0.021$, sample length $l=1 \mathrm{~mm}$ ).

The saturable absorption dynamics is simulated by transitions in a four-level energy diagram. The level system is displayed by the inset in fig. 2. The system of differential equations describing the level populations $N_{i}(i=1$ to 4 ) and the laser propagation is given by [38] 


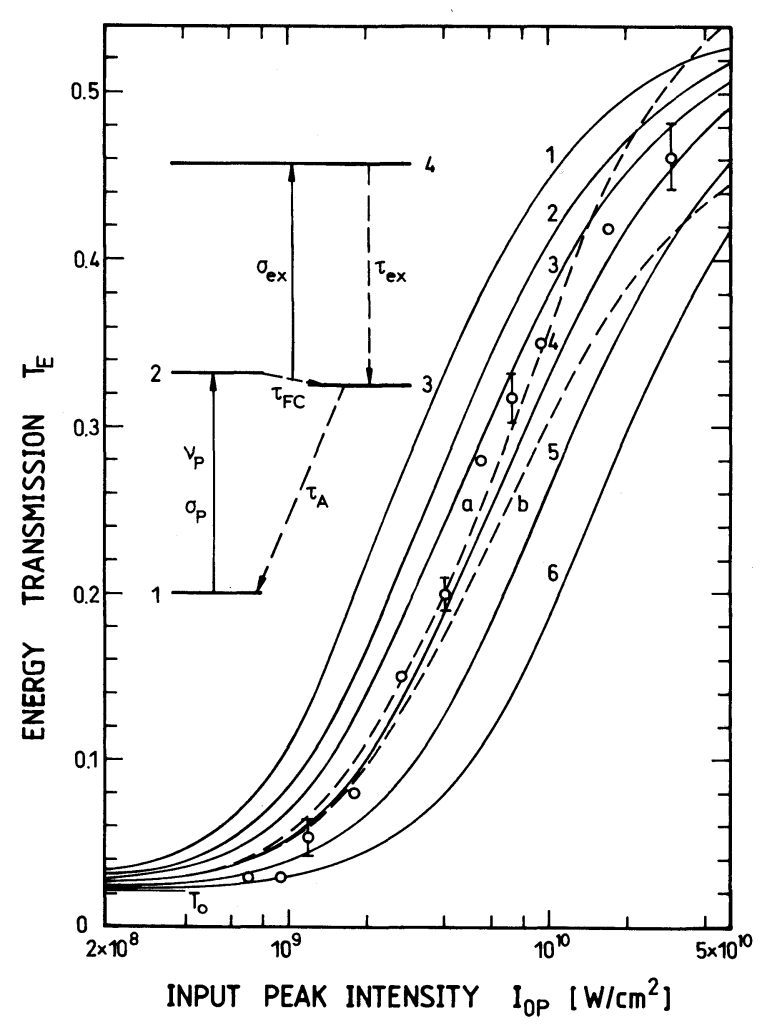

Fig. 2. Pump-pulse energy transmission versus input-peak intensity. Circles represent measured data. The solid curves are calculated for $\sigma_{\mathrm{P}}=1.6 \times 10^{-16} \mathrm{~cm}^{2}[16], \sigma_{\mathrm{ex}}=2.5 \times 10^{-17} \mathrm{~cm}^{2}$, $\tau_{\mathrm{FC}}=0.5 \mathrm{ps}$ (assumed), $\tau_{\mathrm{ex}}=10^{-13} \mathrm{~s}$ (assumed), $\tau_{\mathrm{or}}=150 \mathrm{ps}$ (assumed, no relevance here), $\Delta t_{\mathrm{P}}=4 \mathrm{ps}$, and $T_{0}=0.021$. The absorption recovery times are (1) $\tau_{\mathrm{A}}=\infty$, (2) $\tau_{\mathrm{A}}=8 \mathrm{ps,} \mathrm{(3)} \tau_{\mathrm{A}}=4$ ps, (4) $\tau_{\mathrm{A}}=2 \mathrm{ps}$, (5) $\tau_{\mathrm{A}}=1 \mathrm{ps}$, and (6) $\tau_{\mathrm{A}}=0.5 \mathrm{ps}$. The dashed curve belong to $\tau_{\mathrm{A}}=2 \mathrm{ps}$ and $\sigma_{\mathrm{ex}}=2 \times 10^{-17} \mathrm{~cm}^{2}$ (a) and $\sigma_{\mathrm{ex}}=3 \times 10^{-17} \mathrm{~cm}^{2}(\mathrm{~b})$. The other parameters are the same as for the solid curves. The energy-level diagram applied to the calculations is inserted in the figure.

$$
\begin{gathered}
\frac{\partial N_{1}(\theta)}{\partial t^{\prime}}=-\frac{I_{\mathrm{P}}}{h \nu_{\mathrm{P}}} 3 \sigma_{\mathrm{P}} \cos ^{2} \theta\left[N_{1}(\theta)-N_{2}(\theta)\right] \\
+\frac{N_{2}(\theta)+N_{3}(\theta)}{\tau_{\mathrm{A}}}-\frac{N_{1}(\theta)-\bar{N}_{1}}{\tau_{\text {or }}}
\end{gathered}
$$

$$
\begin{gathered}
\frac{\partial N_{2}(\theta)}{\partial t^{\prime}}=\frac{I_{\mathrm{P}}}{h \nu_{\mathrm{P}}}\left[3 \sigma_{\mathrm{P}} \cos ^{2} \theta\left[N_{1}(\theta)-N_{2}(\theta)\right]\right. \\
\left.-\sigma_{\mathrm{ex}}\left(N_{2}(\theta)-\frac{N_{2}(\theta)}{N_{2}(\theta)+N_{3}(\theta)} N_{4}(\theta)\right)\right] \\
-\left(\frac{1}{\tau_{\mathrm{A}}}+\frac{1}{\tau_{\mathrm{FC}}}\right) N_{2}(\theta)-\frac{N_{2}(\theta)-\bar{N}_{2}}{\tau_{\mathrm{or}}}
\end{gathered}
$$

$\frac{\partial N_{3}(\theta)}{\partial t^{\prime}}$

$$
\begin{aligned}
& =-\frac{I_{\mathrm{P}}}{h \nu_{\mathrm{P}}} \sigma_{\mathrm{ex}}\left(N_{3}(\theta)-\frac{N_{3}(\theta)}{N_{2}(\theta)+N_{3}(\theta)} N_{4}(\theta)\right) \\
& +\frac{N_{2}(\theta)}{\tau_{\mathrm{FC}}}-\frac{N_{3}(\theta)}{\tau_{\mathrm{A}}}+\frac{N_{4}(\theta)}{\tau_{\mathrm{ex}}}-\frac{N_{3}(\theta)-\bar{N}_{3}}{\tau_{\mathrm{or}}}
\end{aligned}
$$

$\frac{\partial N_{4}(\theta)}{\partial t^{\prime}}$

$$
=\frac{I_{\mathrm{P}}}{h \nu_{\mathrm{P}}} \sigma_{\mathrm{ex}}\left[N_{2}(\theta)+N_{3}(\theta)-N_{4}(\theta)\right]-\frac{N_{4}(\theta)}{\tau_{\mathrm{ex}}},
$$

$$
\begin{gathered}
\frac{\partial I_{\mathrm{P}}}{\partial z^{\prime}}=-I_{\mathrm{P}} \int_{0}^{\pi / 2}\left\{3 \sigma _ { \mathrm { P } } \operatorname { c o s } ^ { 2 } \theta \left[N_{1}(\theta)-N\right.\right. \\
\left.+\sigma_{\mathrm{ex}}\left[N_{2}(\theta)+N_{3}(\theta)\right]\right\} \sin \theta \mathrm{d} \theta .
\end{gathered}
$$

The transformation $t^{\prime}=t-c_{0} z / n$ and $z^{\prime}=z$ is used ( $c_{0}$ is the vacuum light velocity, $n$ is the refractive index). The initial conditions are

$$
\begin{aligned}
& N_{1}\left(\theta, t^{\prime}=-\infty, z^{\prime}, r\right)=N_{0}, \\
& N_{2}\left(\theta, t^{\prime}=-\infty, z^{\prime}, r\right)=N_{3}\left(\theta, t^{\prime}=-\infty, z^{\prime}, r\right) \\
& \quad=N_{4}\left(\theta, t^{\prime}=-\infty, z^{\prime}, r\right)=0
\end{aligned}
$$

and

$I_{\mathrm{P}}\left(t^{\prime}, z^{\prime}=0, r\right)=I_{0 \mathrm{P}} \exp \left[-\left(t^{\prime} / t_{\mathrm{P}}\right)^{2}-\left(r / r_{\mathrm{P}}\right)^{2}\right]$.

$t_{\mathrm{P}}=\Delta t_{\mathrm{P}} / 2(\ln 2)^{1 / 2}$ is half the $1 / \mathrm{e}$ width of the pumppulse duration and $r_{\mathrm{P}}$ is the $1 / \mathrm{e}$ width of the laserbeam radius. $N_{0}$ is the total number density of dye molecules. The orientation-averaged number densities of the level populations are

$\bar{N}_{i}=\int_{0}^{\pi / 2} N_{i}(\theta) \sin \theta \mathrm{d} \theta \quad(i=1,2,3)$.

The absorption anisotropy of the electric-dipole in- 
teraction is taken into account for the ground-state absorption $\left(\sigma_{\mathrm{P}}(\theta)=3 \sigma_{\mathrm{P}} \cos ^{2} \theta\right) . \theta$ is the angle between the transition dipole moment for ground-state absorption of the molecules and the direction of the electric field of the pump laser (linearly polarized). $\tau_{\text {or }}$ describes the molecular reorientation of the transition dipole moments. The energy transmission of the pump laser is given by

$T_{\mathrm{E}}=\frac{\int_{0}^{\infty} r \mathrm{~d} r \int_{-\infty}^{\infty} I_{\mathrm{P}}\left(t^{\prime}, z^{\prime}=l, r\right) \mathrm{d} t^{\prime}}{\int_{0}^{\infty} r \mathrm{~d} r \int_{-\infty}^{\infty} I_{\mathrm{P}}\left(t^{\prime}, z^{\prime}=0, r\right) \mathrm{d} t^{\prime}}$,

where $l$ is the sample length.

The curves in fig. 2 are calculated by solving numerically eqs. (1)-(6). The dye parameters used are listed in the figure caption. The best numerical fit to the experimental points corresponds to an excitedstate absorption cross-section of $\sigma_{\mathrm{ex}}=(2.5 \pm 0.3) \times$ $10^{-17} \mathrm{~cm}^{2}\left(\sigma_{\mathrm{ex}} / \sigma_{\mathrm{P}}=0.16 \pm 0.02\right)$ and a ground-state absorption recovery time of $\tau_{\mathrm{A}}=3 \pm 1 \mathrm{ps}$.

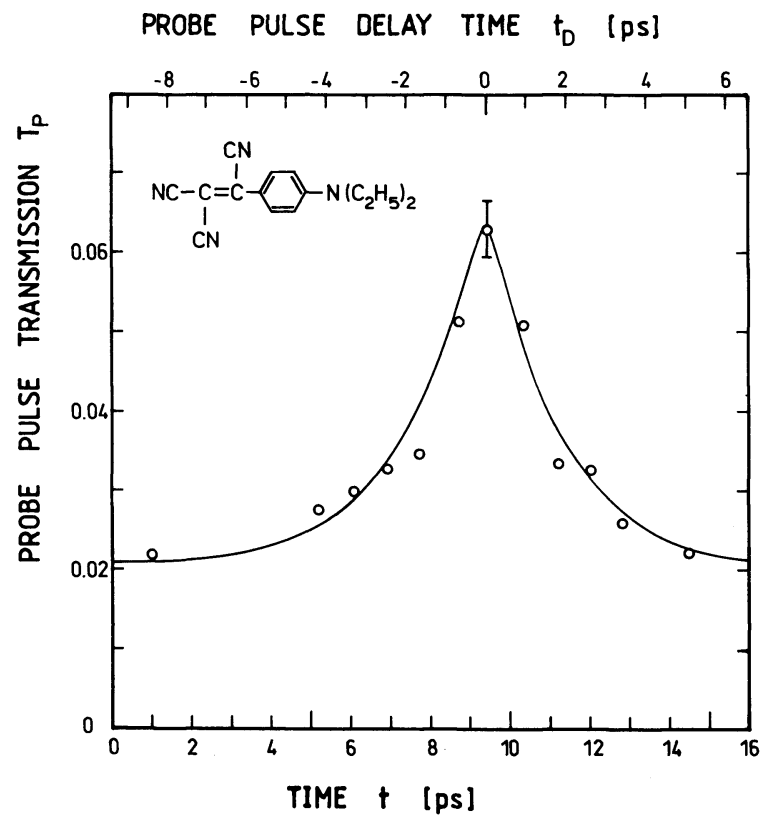

Fig. 3. Probe-pulse energy transmission versus time. Pump-pulse input peak intensity is $I_{0 \mathrm{P}} \pm 2 \times 10^{9} \mathrm{~W} / \mathrm{cm}^{2}$. The small signal transmission is $T_{0}=0.021$. The structural formula of TCVA is inserted in the figure.

\subsection{Probe-pulse transmission}

The temporal position of the probe pulse relative to the pump pulse is varied. The probe-pulse transmission versus time is displayed in fig. 3. Only laser shots with measured pump-pulse peak intensities between $1.5 \times 10^{9}$ and $2.5 \times 10^{9} \mathrm{~W} / \mathrm{cm}^{2}$ were remained for the plot. The probe pulses were attenuated by a factor of 50 compared to the pump pulse.

The probe-pulse transmission curve versus delay time is symmetric relative to the transmission maximum where pump pulse and probe pulse pass simultaneously through the sample (delay time $t_{\mathrm{D}}=0$ ). This symmetric shape indicates that the absorption recovery time $\tau_{\mathrm{A}}$ is shorter than the laser-pulse duration $\Delta t_{\mathrm{P}}$ in agreement with the computer simulations of the pump-pulse transmission (section 3.1).

\section{Discussion}

In ref. [16], the fluorescence quantum efficiency of TCVA in methanol at room temperature was found to be $q \approx 7 \times 10^{-4}$ and a radiative lifetime of the vibrationally relaxated $S_{1}$ state was estimated to be $\tau_{\text {rad }}^{S_{1}} \approx 6 \mathrm{~ns}$. The assumption of $S_{1}-S_{0}$ internal conversion gives a fluorescence lifetime of $\tau_{\mathrm{F}}^{\mathrm{S}_{1}}=q \tau_{\mathrm{rad}}^{\mathrm{S}_{1}} \sim 4$ ps. This value is in good agreement with the groundstate absorption recovery time of $\tau_{\mathrm{A}}=3 \pm 1$ ps determined in this paper. The excited TCVA molecules in methanol relax to the ground state by fast internal conversion at room temperature. Rotational motions of the cyanovinyl group and the diethylamino group seem to be responsible for the fast excited-state deactivation $[10,11,13,16]$. The fast relaxation seems to hinder an efficient TICT state formation.

\section{Conclusions}

The combined measurements of fluorescence quantum efficiencies and absorption recovery times allow the distinction between different relaxation channels of excited molecules. The same information could be obtained by the combined measurements of fluorescence quantum efficiencies and timeresolved fluorescence decays (e.g. streak-camera measurements) [40]. The bleaching behaviour of 
TCVA in methanol at $527 \mathrm{~nm}$ allows the application of the dye as a fast saturable absorber for lasers in this wavelength region (e.g. second-harmonic pulses of $\mathrm{Nd}$ lasers ).

\section{Acknowledgement}

The authors thank the Deutsche Forschungsgemeinschaft for financial support and the Rechenzentrum of the University for the allocation of computer time.

\section{References}

[1] A.T. Peters and M.S. Wild, J. Soc. Dyers Colour 93 (1977) $126,133$.

[2] S.S.M. Hassan, J.M. Abdella and N.E. Nashed, Mikrochim. Acta II (1984) 27.

[3] S.S. Beaven, J.S. Hargreave and D. Phillips, Advan. Photochem. 11 (1979) 207.

[4] N. Taguchi, A. Imai, T. Niwa and Y. Murata, European Part. Appl. EP163145 A2, 4 December 1985.

[5] Mitsubishi Chemical Industries Co. Ltd., Japan. Kokai Tokkyo Koho JP89/7889A2 [84/78895], 7 August 1984.

[6] H.E. Wright and M.A. Berwick, Res. Discl. 158 (1977) 62.

[7] G.R.N. Jones, Nature 235 (1972) 257.

[8] F. Hernadi, Z.N. Nagy, P. Kovacz, Z. Dezsi and T.V. Nagy, Acta Biol. (Budapest) 19 (1968) 257.

[9] N.E. Tonks Jr., Dissertation, University of Georgia, Athens (1986) [Uni. Microfilms Int., Order No. DA8613520].

[10] R.O. Loutfy and K.Y. Law, J. Phys. Chem. 84 (1980) 62.

[11] M.S.A. Abdel-Mottaleb, R.O. Loutfy and R. Lapouyade, J. Photochem. Photobiol. A48 (1989) 87.

[12] R.O. Loutfy, Macromolecules 16 (1983) 678.

[13] R.O. Loutfy, in: Photophysical and photochemical tools in polymer science, ed. M.A. Winnik (Reidel, Dordrecht, $1986)$ p. 429.

[14] J. Aihera, K. Araya and Y. Matsunaga, Bull. Chem. Soc. Japan 54 (1981) 615 .
[15] A.V. Deshpande, A. Beidoun, A. Penzkofer and G. Wagenblast, Chem. Phys. 142 (1990) 123.

[16] A.V. Deshpande, A. Beidoun, A. Penzkofer and G. Wagenblast, Chem. Phys., to be published.

[17] R.O. Loutfy, Pure Appl. Chem. 58 (1986) 1239.

[18] R.O. Loutfy and K.Y. Law, Macromolecules 14 (1981) 587.

[19] K.Y. Law, Chem. Phys. Letters 75 (1980) 545.

[20] K.Y. Law, Photochem. Photobiol. 33 (1981) 799.

[21 ] R.O. Loutfy and R.A. Arnold, J. Phys. Chem. 86 (1982) 4205.

[22] R.O. Loutfy, Macromolecules 14 (1981) 270.

[23] R.O. Loutfy, J. Polym. Sci. Polymer Phys. Ed. 20 (1982) 825.

[24] A. Safarzadeh-Amiri, Can. J. Chem. 62 (1984) 1895.

[25] H.O. Kalinowski and H. Kessler, Topics Stereochem. 7 (1973) 295.

[26] L. Salem, C. Lefrestier, G. Segal and R. Wetmore, J. Am. Chem. Soc. 97 (1975) 479.

[27] G. Orlandi and W. Siebrand, Chem. Phys. Letters 30 (1975) 352.

[28] W.G. Dauben, L. Salem and N.J. Turro, Accounts Chem. Res. 8 (1975) 41.

[29] M. Meyer, J.C. Mialocq and B. Perly, J. Phys. Chem. 94 (1990) 98.

[30] Z.R. Grabowski, K. Rotkiewicz, A. Siemiarczuk, D.L. Cowley and W. Baumann, Nouv. J. Chim. 3 (1979) 443.

[31] W. Rettig, Angew. Chem. Intern. Ed. Eng. 25 (1986) 180.

[32] E.M. Kosower and D. Huppert, Ann. Rev. Phys. Chem. 37 (1986) 127.

[33] E. Lippert, W. Rettig, V. Bonačić-Koutechý, F. Heisel and J.A. Miehe, Advan. Chem. Phys. 68 (1987) 1.

[34] P.F. Barabara and W. Jarzeba, in: Advances in photochemistry, Vol. 15, eds. D.H. Volman, G.S. Hammond and K. Gollnik (Wiley, New York, 1990) p.1.

[35] A. Penzkofer and F. Graf, Opt. Quantum Electron. 17 (1985) 219.

[36] A. Penzkofer and W. Falkenstein, Opt. Commun. 17 (1976) 1.

[37] A. Penzkofer and W. Blau, Opt. Quantum Electron. 15 (1983) 325.

[38] G. Grönninger and A. Penzkofer, Opt. Quantum Electron. 16 (1984) 225.

[39] A. Penzkofer and F. Ossig, Chem. Phys. Letters 154 (1989) 111.

[40] A. Penzkofer and Y. Lu, Chem. Phys. 103 (1986) 399. 